\title{
El periodista educativo y sus circunstancias: lo que el lector no ve
}

The Education Journalist and His Circunstances: What the Reader Can't See Héctor García Barnés ${ }^{1}$

\section{Resumen}

La prensa escrita generalista se ha visto sometida a cambios significativos durante la última década, tanto tecnológicos (la evolución de la prensa de papel a la digital) como económicos (modelos de financiación de la empresa periodística). Una evolución que no ha concluido y que ha condicionado el rol del periodista, tanto del propiamente educativo como de otra índole. Las condiciones del trabajo del profesional raramente son conocidas por el lector cuando se enfrenta a un artículo periodístico sobre educación. Este artículo tiene como objetivo enunciar algunas de las nuevas condiciones del periodista de prensa escrita y analizarlas para entender un poco mejor el proceso de creación de los artículos que llegan a los ojos del lector.

\section{Palabras claves}

Periodismo, educación, periodismo educativo, periodismo digital, modelos de negocio.

\section{Abstract}

The generalist written press has been subject to significant changes during the last decade, both technological (the evolution from printed press to digital) and economic (financial models of journalistic business). An evolution that has not ended and that has conditioned the role of the journalist, educational and otherwise. The conditions of the professional's craft are rarely known by the reader when faced with a newspaper article on education. This article aims to enunciate some of the new conditions of the press journalist and analyze them to understand the hodden process of creation of the articles that reach the eyes of the reader.

\section{Keywords}

Journalism. education, education journalism, digital journalism, business model. 


\section{Introducción}

El artículo periodístico se presenta ante el lector como una obra terminada, completa. A simple vista, y debido que no es explícito, es difícil descubrir aspectos como el tiempo del que ha dispuesto el periodista para investigar y redactar el artículo, su conocimiento sobre el tema, el esfuerzo realizado (o la ausencia de este), así como los posibles intereses tanto del redactor como el medio a la hora de publicar determinado contenido. Tampoco las condiciones materiales en las que se ha realizado, desde el sueldo del redactor hasta su horario laboral, pasando por su formación. Es algo que se ha acentuado en la prensa digital, donde la jerarquización del periódico como producto global y de la página en particular que existían en la prensa de papel han desaparecido. Ahora, cada artículo tiene prácticamente una misma entidad, independientemente de la relevancia que tenga dentro del medio, y corresponde a una URL o dirección específica. Tan solo algunos marcadores externos como la cursiva, para reflejar que se trata de un artículo de opinión y no de información, o la utilización de fotografías más grandes, sirven para diferenciar entre diferentes subgéneros. Por lo demás, todas las noticias, tengan detrás el trabajo de meses o el de apenas media hora, son muy semejantes. Como recuerdan González-Díez, Puebla-Martínez y Pérez Cuadrado, el medio digital ha dejado de ser unidireccional (se pasa de una página a otra hacia delante o hacia atrás) para ser multidireccional y cruzado, lo que también ha provocado que se pierda la antigua jerarquía dentro del periódico (González-Díez et al., 2018:461).

Esto pone de manifiesto la dificultad que tiene el lector a la hora de comprender los condicionantes a los que debe enfrentarse el periodista en su labor diaria. Algunos de los cuales son propios de la profesión; otros tantos han aparecido recientemente, con la evolución de la naturaleza del trabajo originada por cambios de formato, modelo de negocio o composición de las empresas; algunos dependen de las condiciones laborales del trabajador; y, en ciertos casos, dependen propiamente de los temas abordados por el escritor, que tienen sus propios condicionantes. En este análisis abordaremos esos determinantes desde la perspectiva de los cambios más recientes en el caso del periodista educativo que, por las particularidades de su labor, son en parte semejantes a los de otras áreas temáticas, pero que en otros casos, tienen relación con las particularidades de la actualidad educativa tanto local como global.

\section{2. ¿2Existe el periodista educativo?}

La especialización periodística ha sido, durante las últimas décadas (especialmente acentuada a lo largo de los años 90, con el crecimiento de los medios de comunicación), una de las tendencias más acentuadas del periodismo (aunque cabe preguntarse si no se trata de algo consustancial a la labor periodística y que simplemente se expande en épocas de bonanza y se retrae en tiempos de crisis económica), que además le otorgaba una posición privilegiada tanto en el mercado laboral como a la hora de gestionar la información recibida. Como recordaba Fernández Obregón por aquella época, «esta especialización en determinada área lleva a que su opinión sea tomada cada vez más en cuenta, y plantea una de las características fundamentales del periodista especializado: su función de gatekeeper, es decir, el profesional actúa más que nunca como un severo filtro informativo» (Fernández Obregón, 1998).

Entre toda esa especialización, llama la atención que la del periodismo educativo sea particularmente limitado. Raramente existen en España cursos de posgrado que lo aborden, con contadas excepciones como el máster de Periodismo Educativo de la universidad privada CEU San Pablo realizado en colaboración con Grupo Siena, editora del medio especializado Magisterio. En algunos casos, significativamente, el periodismo educativo se entrelaza con otras especializaciones, como ocurre con la asignatura optativa 
«Periodismo especializado en educación y deporte» del grado en Periodismo en la Universidad Complutense de Madrid, en la que se dedican cinco capítulos del temario al deporte y uno a la educación ${ }^{2}$. Como escribía Donaciano Bartolomé Crespo, «el Periodismo educativo, aun reconociendo su utilidad en múltiples aspectos, no deja de sorprender y sobre todo preocupar que tenga un valor de poca significatividad cuantitativa y cualitativamente hablando. Algunos se inclinan a pensar que es porque esta información ni vende, ni tiene demanda, ni mueve dinero» (Bartolomé Crespo, 2005:13).

Este escaso valor aparente tiene su reflejo en el lugar que la educación suele ocupar dentro de un medio, que en muchos casos podría denominarse «transversal». Dicho de otra forma, la educación carece de un espacio propio o regular, como sí ocurre con otra clase de contenidos, y por lo tanto ha de buscar su acomodo en distintas secciones, desde Política hasta Economía pasando por Sociedad, Cultura o secciones de nuevo cuño como Estilos de Vida o equivalentes que, al mismo tiempo, determina el enfoque con el que se va a analizar el hecho educativo. En algunos casos, los artículos de índole educativa aparecen en los suplementos locales de cada medio español, algo lógico teniendo en cuenta tanto que las Comunidades Autónomas tienen un gran número de competencias transferidas sobre educación. Es también una buena muestra de que el sistema educativo tiene una gran proximidad al ciudadano, al contrario de lo que ocurre con la información financiera, por ejemplo. Esta dificultad de encaje se refleja igualmente en la difícil especialización en dicho ámbito por parte de los periodistas. Como recordaba Ramírez de la Piscina, las áreas donde se produce una mayor especialización son Deportes y Economía, y es habitual que la educación caiga en este último grupo (Ramírez de la Piscina, 1999:4).

Entre las dificultades para localizar el periodismo educativo se encuentra, asimismo, que es un amplio subgénero que atañe a muchos actores sociales. Bartolomé Crespo lo define como «aquella actividad periodística que contempla como su objetivo formal el contribuir a que la sociedad sepa y conozca objetivamente, de forma veraz, sin presiones partidistas, ni fobias, ni filas, cuanto a colectivos determinados, a personas singularizadas y a la sociedad en general concierne en los ámbitos de la educación, enseñanza, formación, cultura reglada, no reglada, en todos los niveles académicos y etapas de la vida» (Bartolomé Crespo, 2005:10). Es decir, afecta, virtualmente, a todos los estratos, edades y actores de la sociedad en su conjunto, y es precisamente esa amplitud la que dificulta la especialización, al mismo tiempo que provoca que sea reclamada por otras disciplinas (Política, Economía, incluso Internacional).

\section{Flexibilidad y precarización}

A finales del siglo xx, se produce un creciente proceso de especialización en la prensa española. Como indica Ramírez de la Piscina, «la especialización en los medios ha sido producto de una reordenación funcional y estructural de las empresas, producida por las nuevas exigencias de las audiencias y la creciente competitividad informativa: a mayor ampliación de los contenidos informativos, mayor necesidad de los periodistas especializados» (Ramírez de la Piscina, 1999:5). Eso provocó que apareciesen nuevos medios especializados, suplementos y que el número de páginas de los propios diarios generalistas aumentasen, dando cabida a nuevos contenidos que, hasta cierto punto, satisfacían las necesidades de un mercado publicitario en crecimiento. Esto, no obstante, cambió con la crisis económica global que explotó en 2007 y que se dejó notar sobre todo en la crisis publicitaria de los medios de comunicación que precipitó la del sector (López García, 2015:46-48). Ello provocó el cierre de medios y la reducción de plantillas, que en muchos casos comenzó por aquellas secciones de nuevo cuño o que se percibían más prescindibles,

2 https://www.ucm.es/estudios/grado-periodismo-plan-803611 
de forma que estos fueron asimilados a otras secciones más grandes. Por otra parte, los nuevos medios digitales surgieron con plantillas mucho más pequeñas. En definitiva, si el periodismo educativo fue incluso en los años de bonanza un pequeño reducto, es lógico pensar que la reordenación de las redacciones afectó a dichos contenidos y a los profesionales que se encargaban de ellos.

Si bien la historia de cada medio es diferente, hay una tendencia común a la profesión, especialmente en los medios generalistas, que es una creciente precarización en cuanto a las condiciones materiales de la labor periodística, asociada de forma estrecha a la flexibilidad y la polivalencia que juega en contra de la especialización. López recuerda que «los periodistas de medios convencionales suelen, o al menos solían, tener claramente acotadas sus funciones. Son periodistas asociados con una sección específica (política, deportes, economía, cultura...)» (López García, 2015:63). En el nuevo contexto de incertidumbre empresarial, «esta delimitación de funciones está desapareciendo a marchas forzadas en los cibermedios». Es decir, «la polivalencia laboral es fácilmente traducible en una degradación de las condiciones laborales del periodista. La misma persona que en el pasado se encargaba de una función específica (redactar noticias para una sección del medio de comunicación) ahora ha de desarrollar varias, y ha de ser capaz de completarlas a lo largo de su jornada laboral». En dicho contexto, y entendiendo siempre que hablamos de medios generalistas, la especialización que se había acentuado durante los años anteriores ha terminado derivando en una extrema flexibilidad que dificulta que el periodista que debe cubrir un tema concreto (en este caso, el educativo) pueda conocer en profundidad dicho ámbito. Es más probable, por lo tanto, que esté dedicado a diversas tareas y que su ámbito de actuación sea mucho mayor (por lo que su conocimiento también será más superficial) y que debido a que la actualidad educativa es un tanto «estacional» (con picos en las épocas de exámenes, matriculación o fin de curso), se dedique a otros menesteres en momentos de menos productividad con el objetivo de optimizar recursos.

Pero la flexibilidad y la precarización no solo afectan a la especialización, sino también a las condiciones de trabajo de los periodistas, que han de hacer frente a una mayor carta de trabajo a medida que el número de periodistas en plantilla se ha reducido. Según estima la Asociación de la Prensa de Madrid, entre 2008 y 2013 se destruyeron en el sector alrededor de 11151 puestos de empleo ${ }^{3}$. Es decir, la optimización del tiempo y el esfuerzo obligadamente causan que el periodista disponga de un mayor margen temporal para investigar sobre un tema o localizar nuevas fuentes, lo que ayudaría a conferir una mayor profundidad a sus artículos. No es algo propio únicamente de los medios de papel, sino también de los digitales, que aparecieron a medida que los primeros declinaban. López García recuerda que la mayoría de medios de Internet se organizan con «un perfil bajo en lo económico»: «El funcionamiento óptimo de un cibermedio requiere aligerarlo en lo posible, y combinar una redacción pequeña con el recurso a un plantel amplio de colaboradores; unos y otros, con salarios modestos» (López García, 2015:16). Es factor que hay que tener en cuenta a la hora de reflexionar sobre la remuneración de los profesionales que se encargan de los temas educativos. Ya que, en principio, los periodistas más especializados, por serlo, pueden exigir unas mejores condiciones (tanto económicas como de ritmo de trabajo), muchos medios se decantan por profesionales «junior» o de perfil más bajo para cubrir esos contenidos que, como la Educación, -y según el valor que le dé cada uno de los medios- son considerados secundarios.

La precariedad económica, por otra parte, es determinante en la actitud del periodista en su día a día. No solo por la más o menos inconsciente adaptación de su tiempo y esfuerzo al sueldo percibido, sino

3 https://www.apmadrid.es/informe-de-la-profesion-periodistica-2013-11151-empleos-perdidos-y-284-medios-cerrados-desde-2008/ 
también por su posición ante las presiones externas, sean de las fuentes con las que trabaja como los intereses de sus superiores. Es, en otras palabras, una manera de convertir en «dócil» al trabajador, que al ver su puesto amenazado, es más propenso a ceder a las presiones de superiores, de organismos oficiales o de empresas privadas: «De los problemas planteados, la cuestión que más preocupa a todos los periodistas encuestados es la precariedad y la inseguridad laboral. El segundo aspecto que suscita más inquietud es el predominio de los intereses económicos y políticos sobre los periodísticos» (Figueras-Maz et al., 2012: 72). Son dificultades propias a toda la profesión periodística, pero que puede notarse aún más en la información educativa, como hemos visto poco consolidada incluso en el período de especialización. Cabe recordar, igualmente, que la precariedad se traduce en la dificultad para identificarse con la empresa para la que se trabaja. Además, han desaparecido figuras de radical importancia en el control de calidad de los medios como correctores, editores o fact-checkers.

\section{Un nuevo modelo de negocio}

Tradicionalmente, la principal fuente de financiación de los medios generalistas españoles fue la publicidad. Durante los años de la crisis económica se produjo un notable descenso en la inversión publicitaria que provocó tanto que la lucha por esta entre medio fuese más dura como que se buscasen otras fuentes alternativas de financiación y modelos de negocios que siguiesen permitiendo hacer viables los medios escritos. Aunque la inversión en publicidad se ha reducido en los medios convencionales de papel y aumentado en los medios digitales, esta sigue siendo mucho más baja que lo que lo era en los momentos de esplendor de la prensa escrita, por lo que el descenso no ha disco compensado. Según los datos del Observatorio de la Prensa Diaria de la AMI (Asociación de Medios de Información) ${ }^{4}$, en 2017 la prensa escrita ingresó 469,1 millones por publicidad, mientras que la de los medios digitales se quedaba en 194,8 millones. En definitiva, y a pesar de su reducción, la prensa de papel sigue acaparando gran parte del pastel publicitario. Como añade López García (2015:48), «un lector en Internet se paga diez o veinte veces peor, en términos publicitarios, que el mismo lector en la prensa, o un espectador de televisión».

A pesar de ello, el modelo predominante en los medios digitales emergentes ha seguido siendo el de la publicidad, heredado de la prensa escrita. En muchos casos, se trata de una publicidad display (como el de los banners) que es particularmente barata y que en los anunciantes deciden invertir en función de la cantidad de tráfico generado por el medio, el equivalente digital del número de periódicos vendidos en prensa escrita. En otras palabras, cuanto más tráfico se genera, más posibilidades hay de captar a los mejores anunciantes, aunque el mercado esté cada vez más dirigiéndose a la segmentación de audiencias. Por lo tanto, la generación de tráfico, entendida como la cantidad usuarios únicos que leen el medio en su conjunto (aunque, en muchos casos, no hay entrado nunca a la homepage del medio, algo imposible en la prensa de papel), ha sido, por lo tanto, una de las obsesiones de los medios de comunicación durante el último decenio. Hay que tener presente, en este punto, que cuando alguien adquiría un periódico, era casi imposible conocer con exactitud qué noticias preferían los lectores, cuáles leían y cuáles dejaban a la mitad. Ahora, gracias a herramientas de analítica web como Google Analytics, que ofrece información agrupada del tráfico que llega a los sitios y que es utilizado como medición referencia en el marketing, o herramientas de visitas a tiempo real como Chartbeat, es posible saber con bastante aproximación dichos datos, pero también la cantidad de tráfico generado por cada redactor, propiciando que el medio ya no se entienda como un todo y favoreciendo que cada uno de sus periodistas se aísle aún más, asediado por la presión de las métricas.

4 https://www.elespanol.com/economia/medios/20180404/publicidad-diarios-papel-paga-veces-cara-digitales/297221237_0.html 
Ello ha influido sobremanera en, ante todo, la elección de temas por parte del periodista educativo precario para conseguir encontrar enfoques «que se lean», descartando otros que pueden ser más arduos, menos atractivos para el gran público o de alcance más limitado, aunque no necesariamente menos interesantes. Como resume López García (2015:116), «esto está llevando, en la mayoría de los sitios web, a definir sus estrategias según este criterio, y a cuantificarlo todo: no solo la audiencia, también las noticias y los periodistas (se valoran las noticias según los impactos, los periodistas según sus seguidores en las redes sociales, etc.)». Es una nueva forma de criba temática que hace que se relativicen determinados enfoques y contenidos en favor de otros. En ese sentido, los titulares juegan un papel de radical importancia a la hora de centrar la atención del lector, mientras que en los medios tradicionales entraban en juego otros factores como las fotografías o la colocación en la página. Además, la velocidad de trabajo es esencial, ya que publicar antes que la competencia es un factor decisivo a la hora de posicionar una noticia en un buscador como Google, lo que multiplica las posibilidades de que un mayor número de lectores lleguen a él. Esto hace que en muchos casos llegue a primar la rapidez incluso por encima de aspectos como la corrección gramatical y ortográfica, ya que la edición digital permite corregir el artículo una vez esté publicado, algo que no ocurría en el papel.

Aunque debido a la opacidad de los medios y a lo reciente de este sistema no existe aún una taxonomía de qué «se lee» en temática educativa, se puede intentar seguir el camino opuesto y realizar una inferencia examinando cuáles son las temáticas que abundan en tiempos recientes en la prensa digital, más allá de los consabidos acontecimientos educativos de los que debe dar cuenta un medio generalista (legislación, huelgas, etc.). Entre ellas quizá el caso paradigmático sea el 'ranking', entendido en el sentido amplio más amplio, con el informe PISA en el que profundizaremos más tarde, a la cabeza. Pero también es un buen ejemplo contenidos en principio no periodísticos, pero sí publicados por medios de comunicación, como el listado de Los 100 mejores colegios de España ${ }^{5}$ realizado anualmente por El Mundo desde hace 19 ediciones y que ha terminado convirtiéndose en el 'ranking' de referencia. Este contenido que no encaja en ninguno de los géneros tradicionales señala de forma elocuente una de las peculiaridades que ha adoptado el periodismo educativo durante los últimos tiempos, que es constituirse como una guía de consumo para estudiantes y padres a la hora de orientarse en la elección de un centro y que tratan a estos como clientes. Los 'rankings' de colegios y universidades son un subgénero cada vez más popular a nivel global, y muchos grandes medios tienen el suyo propio, o utilizan los de otras organizaciones como fuente ${ }^{6}$ en caso de que no haya recursos suficientes. Otro buen ejemplo es la Clasificación académica de universidades de THE (Times Higher Education), uno de los 'rankings' globales referencia y que lleva el sello de la principal publicación periodística sobre Educación superior del Reino Unido.

El periodista educativo raramente participa en la elaboración de estos «rankings» ni mucho menos influye en sus criterios, aunque se haga eco de ellos o los utilice como fuentes para sus artículos. No obstante, estas sí determinan gran parte de la visión del mundo educativo que tienen tanto los profesionales como los lectores. Es lo que ocurre con otro de los temas que abundan en el periodismo educativo contemporáneo: el análisis de sistemas educativos lejanos que sirven como ejemplo al nuestro, que suelen salir favorecidos en los 'rankings' y cuya fama se debe a la aparición en estos, así como el retrato de colegios 'milagro' que han alcanzado un éxito educativo óptimo. Es lo que ocurre tradicionalmente,

\footnotetext{
5 http://www.elmundo.es/mejores-colegios.html Llamativamente, el contenido se llama Los 100 mejores colegios de España sin aludir a que en él no participan los colegios públicos, tan solo los privados.

6 https://www.elconfidencial.com/alma-corazon-vida/2018-05-09/ranking-cyd-2018-universidades-cataluna_1560952/
} 
por ejemplo, con Finlandia o los países asiáticos. En algunos casos, estos contenidos son patrocinados y aparecen en secciones especiales, como ocurre con un vídeo-reportaje $\mathrm{p}^{7}$ publicado en el suplemento de El País Aprendemos Juntos sobre la escuela pública portuguesa y que lleva el sello de la entidad financiera BBVA, así como el de la editorial Santillana en el cintillo que preside la página.

Conviene llamar la atención sobre nuevas formas publicitarias que están emergiendo y que, por ahora, ofrecen posibilidades tanto para los medios como para los anunciantes que hasta hace no tanto tiempo no eran pensables. Es el caso del branded content, descrito como «la creación de contenidos por parte de la marca orientados a mantener conversaciones con el nuevo usuario», siendo el objetivo último de este formato «la transmisión de valores en un doble sentido: de la marca al contenido y del contenido a la marca». (Díaz Anichtchenko y Breva Franch, 2017:107). Se trata de una fórmula semejante a la del patrocinio, con la diferencia de que el segundo «suministra fondos, recursos y/o servicios a un determinado activo» y el primero «crea o financia contenidos» (Díaz Anichtchenko y Breva Franch, 2017:110). Es decir, cada vez es más común que el periodista de un medio forme parte de grupos dedicados exclusivamente a la elaboración de estos contenidos de marca que son encargados por empresas de muy diversa índole a través de un procedimiento similar al publicitario.

Estos modelos dan un mayor poder a las empresas anunciantes, que pueden terminar determinando contenidos en función de sus intereses: «Un medio de comunicación exclusivamente dependiente de la publicidad pierde autonomía para analizar la realidad» (López García, 2015: 76). Además, obliga a las empresas a competir duramente por una mayor cantidad de tráfico; si bien es cierto que, en teoría, un medio de baja calidad atraerá poca publicidad, en la práctica los nuevos medios digitales tienden a buscar nuevos anunciantes atraídos a través de sus cifras. Frente a ello está apareciendo una nueva fórmula poco explotada en España salvo en medios contados como Infolibre, que por lo general se han saldado con fracasos: la del muro de pago o pay-wall, es decir, pagar por leer parte de un medio o el medio completo. Como recuerdan García-Santamaría, Pérez-Serrano y Maestro-Espínola (2016:396), «después de un período de tiempo en el que no había ninguna corriente favorable a instalar sistemas de suscripción (pay walls o "muros de pago"), se observa cómo a partir de 2014 la mayoría de grandes diarios han adoptado estrategias de affinity clubs (clubs de suscriptores o clubs de lectores)». Una apuesta por la calidad que ha sido liderada por uno de los grandes medios de referencia globales, The New York Times, que ha conseguido demostrar que es un modelo no solo viable, sino rentable. Además, uno en el que ya no se depende de los anunciantes y sus caprichos, sino sobre todo, del público que es quien juzga atractivos (o no) los contenidos ofertados y que, por esa manera, puede dar más valor a un contenido especializado como el educativo. Sin embargo, es posible que el impacto de esta fórmula sea moderada en otra clase de medios, apenas de un 10\% del total (Myllylahti, 2014).

Por último, llama la atención que, a medida que las secciones relacionadas con Estilos de Vida se han hecho hueco en los periódicos, hayan aparecido contenidos prácticos destinados directamente a un destinatario que es el propio estudiante. Se trata, por ejemplo, de los artículos que tienen como objetivo ayudarle en el estudio desde una perspectiva psicológica o aquellos que le presentan las posibilidades de futuro de la carrera escogida. Una vez más, prima la visión del estudiante como cliente del sistema educativo. Una visión de la educación que entronca con lo que Liria, García y Fernández denominan «El

\footnotetext{
7 https://aprendemosjuntos.elpais.com/especial/es-portugal-la-nueva-finlandia-de-la-educacion-escuela-publica-carcavelos/

8 https://extraconfidencial.com/noticias/los-muros-de-pago-en-internet-no-funcionan-en-nuestro-pais-el-mundo-el-espanol-e-infolibre-fracasan-en-su-intentode-aumentar-sus-ingresos-por-esta-via/
} 
gran mercado de la educación»: «La escuela pública se encuentra ahora mismo en el centro de un huracán neoliberal que amenaza con arrasarla por completo y convertirla en otra cosa. Hay toda una serie de instituciones al servicio de los intereses del capital internacional empeñadas en convertir todo el sistema educativo en un negocio rentable. A nivel mundial, la educación representa un presupuesto anual de más de un billón de dólares, unos 50 millones de trabajadores en el sector y una clientela potencial de mil millones de alumnos y universitarios» (Fernández Liria et al., 2017:71) Una reflexión que nos lleva al siguiente punto: la naturaleza de las fuentes de las que el periodista educativo obtiene su información y cómo la evolución de los modelos de negocio y de las condiciones del trabajo periodístico han cambiado su naturaleza.

\section{Fuentes... ¿̇qué fuentes?}

A las fuentes, sean estas de la índole que sean, son la materia prima del periodismo, y su selección termina conformando el eje vertebrador de los reportajes informativos. Uno de los riesgos más evidentes de tener que trabajar a máxima velocidad y elaborar rápidamente los artículos -los tiempos en la prensa digital ya no están marcados por la edición del día siguiente, sino que pueden ser automáticamente publicadoses la dificultad para contrastar informaciones, por lo que en muchos casos se suele dar como válida la primera fuente, que por lo general (y especialmente en el ámbito educativo) suele ser muy interesada. Bartolomé Crespo recordaba que «las fuentes de la información en la Educación resultan un elemento muy importante, puesto que la sociedad necesita y debe conocer el funcionamiento y los entresijos de un mundo tan complejo y tan burocratizado como es el ámbito educativo» (Bartolomé Crespo, 1995:137). El autor recordaba que las fuentes se habían multiplicado durante los años anteriores a la publicación de su trabajo, y ya señalaba una particular carencia de los periodistas educativos. Se trata, en primer lugar, de una «carencia de fuentes» que se reflejaba en que «en los medios de comunicación aparecen casi siempre las mismas y que son poco variadas: Ministerio de Educación, Cultura y Deportes, algunas sindicales, asociaciones de familias y agencias generalistas de información como pueden ser EFE y Europa Press》 (Bartolomé Crespo, 1995:138). Por otra parte, aludía a una limitación de los temas tramados, generalmente y como ya hemos citado, estacionales: «Se aprecia una actitud informativa de esperar, no de buscar. Y en el ámbito educativo acontecen múltiples eventos de gran importancia e interés informativo y para el público».

Es posible, dada la situación, que esta última tendencia se haya exacerbado en los últimos años. Dado que los profesionales dedicados al periodismo educativo son pocos o, simplemente, se trata de profesionales de otras áreas que ocasionalmente abordan la cuestión educativa, es probable que el periodismo educativo sea reactivo, es decir, se realice tan solo ocasionalmente ante una circunstancia que tiene interés general. Al mismo tiempo, esta reactividad de los contenidos provoca que muchos de ellos vengan dados por los actores implicados y no que sean producto de una larga investigación del periodista o de una idea que emerge de este. Hemos visto ya como las empresas educativas de índole privada (colegios, fundaciones, etc.) pueden determinar los contenidos a partir del patrocinio o del branded content. Pero también es posible que lo hagan sugiriendo enfoques o contenidos al periodista educativo (o no) precario que, siempre ávido de temas (y de artículos «que se lean» puede terminar dándolos por buenos tal cual.

Es revelador que en la minuciosa clasificación realizada por Bartolomé Crespo sobre las fuentes educativas apenas tengan peso estos nuevos actores que tanta relevancia han adquirido durante los últimos tiempos, y prácticamente se de por hecho que no se trata de fuentes válidas. Como recuerda Mayoral 
Sánchez, «sabemos que las fuentes han trazado un minucioso plan para difundir su versión de la realidad: por eso hay miles y miles de gabinetes de prensa por doquier. No sabemos, en cambio, si el periodismo dispone de un plan de defensa para mantener su relato a salvo de versiones conscientemente deformadas de la realidad» (Mayoral Sánchez, 2005:94). Ya en 2005, llegaba a la conclusión en su análisis de 6158 textos que la media de fuentes por texto era de 1,33. El 32,80\% de las cuales no aparecían debidamente identificadas, y en el 46,5\% de casos no tenían ninguna réplica, aunque haga referencia a los intereses particulares de la fuente. Es presumible pensar que el porcentaje será aún menor en los últimos años, a medida que el periodismo digital y la desaparición de figuras de control como el editor han relajado las costumbres, imponiéndose el del tráfico y la rentabilidad como principales criterios.

En ese sentido es probable que gran parte de la información educativa se encuentre en sintonía con lo que se ha dado en llamar la «mercantilización de la educación», que ya no es vista como un servicio público sino como un producto en el cual los educandos son sus clientes. Como aseguraba Nico Hirtt, es «una nueva etapa histórica en un movimiento que se extiende durante más de un siglo: el deslizamiento progresivo de la Escuela, desde la esfera ideológico-política hacia la esfera económica; de la "superestructura" hacia la "infraestructura", podríamos decir en jerga marxista» (Hirtt, 2004:2). Ello también se refleja en los contenidos del periodismo educativo, que se sitúan entre dar voz a los poderes públicos, como el Ministerio de Educación, y hacerlo con las empresas privadas y otras organizaciones de índole económica cuyos objetivos no terminar de quedar claros al no estar identificados en el texto, al menos para el lector. Las prisas y las condiciones precarias de trabajo, por otra parte, juegan en contra del criterio del periodista.

\section{El caso de PISA: un ejemplo elocuente}

De todas las informaciones relacionadas con el sistema educativo, pocas pueden compararse con el interés que suscita el examen PISA, el estudio llevado a cabo por la OCDE para medir las capacidades de los estudiantes de quince años en lectura, matemáticas y comprensión de la ciencia ${ }^{9}$. Es uno de los contados momentos en los que la información educativa salta a las aperturas de los medios, sean digitales o en papel. Es lo que ocurrió, por ejemplo, con medios nativos de este formato como El Confidencial o El Español, que dedicaron especiales a este acontecimiento en los que, por una vez, a la noticia principal se añadieron otras informaciones adicionales. De ahí que el tratamiento de la información de este informe sea especialmente revelador de las tendencias en la prensa española.

Una investigación de González-Mayorga, Vidal y Vieira (2017) sobre el impacto de PISA en la prensa escrita sobre una muestra de 778 noticias publicadas en el diario El País revelaba algunos datos llamativos. Para empezar, el $71 \%$ de los artículos relacionados con el tema fueron publicados por periodistas, un $58,1 \%$ de los cuales no estaban especializados en educación lo que muestra, una vez más, que incluso en el caso de un evento tan en principio relevante como la publicación de este informe ni siquiera se cuenta con profesionales especializados. Un 9,8\% estaba firmado por profesores universitarios mientras que un 7,6\% pertenecía a otros ámbitos, como escritores, politólogos, bibliotecarios, abogados, etc. De los artículos analizados, 99 llevaban la misma firma, la de J. A. Aunión, responsable de Educación de El País. Además, se producía una llamativa diferenciación: mientras que la información es escrita por periodistas, la opinión suele ser escrita por profesionales de otros ámbitos ajenos al propiamente educativo, lo que lleva a pensar que raramente son los expertos del propio ámbito los que gozan de espacio para opinar en

9 https://nces.ed.gov/surveys/pisa/ 
los medios de comunicación generalistas. Los profesores de Primaria y Secundaria (las etapas analizadas en PISA) apenas escriben un 3,9\% del total de artículos. Cabe destacar que el período analizado es amplio, y abarca tanto la crisis como la inmediata poscrisis, de 2001 a 2013. Una amplia mayoría de las publicaciones relacionadas con este tema eran noticias (45,1\%), seguidos por artículos de opinión (18,9\%) y reportajes $(12,6 \%)$.

Es elocuente, por lo tanto, que el informe PISA se tome ante todo como material de noticia y no tanto de reflexión, puesto en contexto, a través de otros formatos como el reportaje. Ello se refuerza por las fuentes que suelen ser utilizadas para realizar las informaciones, y que por lo general provienen del ámbito político. En el «ranking» de fuentes consultadas se encuentran estas fuentes institucionales como la del Ministerio de Educación (39,1\%), seguidas por miembros de la OCDE (19,6\%) y otras entidades $(15,2 \%)$. El profesorado de Secundaria representa un 10,9\% del total. Unos datos que muestran una llamativa tendencia de la información educativa a dar voz a discursos políticos o económicos (conviene no perder de vista que la propia OCDE, Organización para la Cooperación y el Desarrollo Económicos es, como su nombre indica, un organismo de ámbito económico). Además, los autores llaman la atención sobre otro dato significativo: «La poca incidencia de artículos de opinión del lector, que puede deberse al desinterés por parte de los lectores por los asuntos relacionados con PISA o, por el contrario, a que la opinión de la ciudadanía sobre este tema no interesa a los medios» (González-Mayorga et al., 2017: 5). ¿La pescadilla que se muerde la cola? Estos concluyen que «incluso un estudio con el peso y la trascendencia de PISA puede presentar limitaciones metodológicas, por lo que es necesario que los debates se alimenten de diversas fuentes de información y, en todo caso, se realicen con un uso más prudente de las mismas» (González-Mayorga et al., 2017:15), lo que es cada vez más difícil a medida que la exigencia de actuar con premura aumenta.

Cabe recordar en este punto que la información del informe PISA proporcionada a los periodistas se administra bajo embargo, es decir, estos reciben la información un día antes de hacerse públicos con el compromiso de no publicar nada hasta la hora prevista, una herramienta promocional que, como ha recordado Kiernan (2006) «sirve para controlar la forma en la que los contenidos periodísticos se divulgan y para promover importantes historias científicas». Este sistema, no obstante, «promueve que los periodistas trabajen como un pack proporcionando la misma información y los mismos plazos a aquellos que están suscritos al sistema de embargos». Bajo la apariencia de libertad que en teoría promueve este sistema, ya que en principio da margen al redactor a profundizar en los datos y llegar a sus propias conclusiones, Kiernan considera que en realidad agudiza la tendencia de los periodistas a hacer un trabajo similar al de su competencia inmediata. Algo agudizado por el funcionamiento de las redacciones, donde se imponen determinados enfoques convencionales por encima de otros más heterodoxos.

El caso del informe PISA como producto periodístico estrella es particularmente revelador, ya que señala unas cuantas de las peligrosas tendencias que caracterizan al periodismo moderno: informaciones elaboradas por redactores no especializados, utilización de fuentes institucionales y gubernamentales, homogeneidad en los enfoques, información administrada a discreción por agencias de índole económica, escasa presencia de los profesionales de la educación en los reportajes sobre este tema y superficialidad e inmediatez por encima de la reflexión.

\section{Conclusión}

El rol y las condiciones de los periodistas han cambiado durante los últimos años de una manera que no 
siempre resulta clara para el lector, que desconoce los condicionantes ocultos que determinan la labor de este. Entre todas las figuras especializadas, es probable que el periodista educativo sea uno de los que peor lo han tenido; eso en caso de que podamos hablar de periodista educativo, y no de simple reportero al que, por cuestiones coyunturales, le toca cubrir información educativa. En este artículo hemos querido enumerar algunos de estos cambios y analizar de qué forma han podido afectar a la labor diaria del periodista, y por extensión, al resultado de su trabajo, tanto en términos de calidad (condicionado por cuestiones de tiempo, dinero o formación) como a la hora de filtrar la información que recibe, y que tanto una vez más por una cuestión temporal como por otros factores como el acceso rápido a la información termina dando prevalencia a unas visiones y enfoques por encima de otras, así como primando las voces de más fácil acceso y mayor poder por encima de las minoritarias. Entre todas ellas, quizá sobresalga la influencia creciente que en los medios de comunicación tiene el sector privado y las instituciones de índole económica, cuyos intereses particulares no siempre quedan claros en las informaciones y opiniones publicadas por los profesionales del periodismo educativo.

\section{Referencias bibliográficas}

Díaz Anichtchenko, Cristina y Breva Franch, Eva (2017): "El patrocinio en la era del branded content". Pensar la Publicidad, 11, 101-120.

Bartolomé Crespo, Donaciano (2005). Periodismo educativo. Madrid: Editorial Síntesis.

Fernández Liria, Carlos; García Fernández, Olga; Galindo Fernández, Enrique (2017). Escuela o barbarie. Entre el neoliberalismo salvaje y el delirio de la izquierda. Madrid: Akal.

Fernández Obregón, Francisco Javier (1998). “Especialización, futuro del periodismo”. Revista latina de comunicación social, 7. Recuperado el 30 de julio de 2018 de: https://www.ull.es/publicaciones/latina/ a/70obr.htm

Figueras-Maz, Mònica; Mauri-Ríos, Marcel; Alsius-Clavera, Salvador; Salgado-De-Dios, Francesc (2012): "La precariedad te hace dócil. Problemas que afectan a la profesión periodística". El profesional de la información, 21 (1), 70-75. DOI: https://doi.org/10.3145/epi.2012.ene.09

García-Santamaría, José-Vicente; Pérez-Serrano, María-José; Maestro-Espínola, Lidia (2016): “Los clubs de suscriptores como nuevo modelo de financiación de la prensa española". Elprofesional de la información, 25 (3), 395-403. DOI: https:/ / doi.org/10.3145/epi.2016.may.09

González-Díez, Laura; Puebla-Martínez, Belén y Pérez-Cuadrado, Pedro (2018): "De la maquetación a la narrativa transmedia: una revisión del concepto de 'diseño de la información periodística"'. Palabra Clave, 21 (2), 445-468. DOI: https://doi.org/10.5294/pacla.2018.21.2.8

González-Mayorga, Héctor; Vidal, Javier; Vieira, María José (2017): “El impacto del Informe PISA en la sociedad española: el caso de la prensa escrita”. RELIEVE, 23 (1), art. 3. DOI: https://doi. org/10.7203/relieve.23.1.9015

Kiernan, Vincent (2006). Embargoed Science. Chicago: University of Illinois Press.

López García, Guillermo (2015). Periodismo digital. Redes, audiencias y modelos de negocio. Salamanca: Comunicación Social. Ediciones y publicaciones. 
Mayoral Sánchez, Javier (2005): "Fuentes de información y credibilidad periodística”. Estudios sobre el mensaje periodístico, 11, 93-102.

Myllylahti, Merja (2014): "Newspaper Paywalls—the Hype and the Reality". Digital Journalism, 2 (2), 179194, DOI: https://doi.org/10.1080/21670811.2013.813214

Ramírez de la Piscina, Txema (1999): "Realidad y utopía de la especialización en el periodismo”. Revista de estudios de comunicación, 4 (6).

\section{Nota biográfica}

Héctor García Barnés es Licenciado en Comunicación Audiovisual por la Universidad Complutense de Madrid, obtuvo su Diploma de Estudios Avanzados por el trabajo El bosque: cine y relato tras el 11 de septiembre y posee un Máster en Formación del Profesorado de Educación Secundaria por la Universidad Rey Juan Carlos. Trabaja como periodista en El Confidencial y es autor del libro La ley de las aulas. La educación española desde Franco hasta Wert (Editorial UOC, 2016). 